East African Medical Journal Vol. 85 No. 4 April 2009

EVALUATION OF SUSPECTED RENAL COLIC PATIENTS WITH UNENHANCED LOW-DOSE MULTI-DETECTOR COMPUTED TOMOGRAPHY

N. Twahirwa, MBChB (Mak), Radiology Resident and J. Rees, MBChB (Edin), FRCR, FRCP (C), Professor of Radiology / Director PGME, Aga Khan University, Nairobi, P.O. Box 30270-00100, Nairobi, Kenya

Request for reprints to: Dr. N. Twahirwa, Radiology Department, Aga Khan University, Nairobi, P.O. Box 30270-00100, Nairobi, Kenya

\title{
EVALUATION OF SUSPECTED RENAL COLIC PATIENTS WITH UNENHANCED LOW-DOSE MULTI-DETECTOR COMPUTED TOMOGRAPHY
}

\author{
N. TWAHIRWA and J. REES
}

\begin{abstract}
Objectives: To determine the proportion of correctly diagnosed ureteric calculi and alternate diagnoses using unenhanced low dose multidetector CT KUB.

Design: Descriptive - prospective study.

Setting: Aga Khan University Hospital, Nairobi between November 2006 and October 2007.

Subjects: One hundred and four patients underwent low dose multi-detector computed tomography (MDCT) KUB.

Results: Ninety (47 males and 43 females with age range of $17-79$ years, mean 40 years) were included in the statistical analysis while 14 were excluded. Eighteen out of ninety $(20 \%)$ were classified as definitely having ureteric calculi on the composite reference. Among the 18, 17 were correctly identified on unenhanced low dose MDCT, giving a proportion of (17/18) $94.4 \%$, Pearson Correlation of 0.898 and Fishers exact test significance $<0.001 .29 / 90(32 \%)$ had alternative diagnosis and $42 / 90(47 \%)$ had no abnormality detected on unenhanced low dose MDCT.

Conclusion: This study shows that, unenhanced low dose MDCT KUB can be effectively used for evaluation of suspected renal colic patients as demonstrated by the statistically significant correctly detected ureteric calculi.
\end{abstract}

\section{INTRODUCTION}

Acute renal colic is probably one of the most excruciatingly painful event a person can endure. (1). The single most common cause of acute renal colic is an obstructive ureteric calculus. However it's important to note that non-calculus and nongenitourinary conditions can present in a similar manner. Since renal colic presents as an emergency, a rapid diagnostic tool is required in such situations. Currently CT KUB has become the clear test of choice for imaging patients with suspected renal colic for a variety of reasons including its speed, non utilisation of contrast, high accuracy for diagnosis or exclusion of stone and determination of stone burden, size and location; assessment of obstructive effects of the stone; identification of significant alternative and additional diagnoses; utility in guiding appropriate patient management (2-4).

Unenhanced helical CT (CT KUB), originally described by Smith et al (5) in 1995, has been shown to be more accurate compared to abdominal radiography $(6,7)$, ultrasound and has replaced excretory urography in the detection of urinary tract calculi in many situations (8). Therefore it has gained widespread acceptance among radiologists, emergency department physicians, and urologists.

However because of the associated radiation dose, various protocols have been studied in an attempt to reduce this dose and found to be equally accurate $(2,3,4,9)$. Recent research focus has been establishing appropriate low dose technique. The purpose of this study was therefore to evaluate the diagnostic performance of a low radiation protocol in the patient population referred to our department, by comparing low dose MDCT KUB findings and clinical outcome(s).

\section{MATERIALS AND METHODS}

Study design and number of subjects: This was a descriptive cross-sectional study, where the unenhanced low dose MDCT findings were compared with the clinical laboratory surgical findings mentioned below as reference. Data was collected over a period of one year (November 2006 to October 2007). The Aga Khan University Research and Ethics Committee approved this study. 
Inclusion criteria: Study subjects included all patients with renal colic and referred to the radiology department for CT KUB and prospectively followed up for eventual outcomes.

Exclusion criteria: Patients excluded from analysis were mainly those lost to follow-up.

CTexamination and interpretation: There was no need of patient preparation apart from filling their bladder just before CT examination. Unenhanced scans meant no oral or in travenous contrast administered, which is also a key advantage over IVU. These scans were done using GE Light speed MDCT in supine position covering the region between the lung bases and pubic symphysis. Acquisition parameters included $120 \mathrm{kVp}$ and tube current reduced from 200 $\mathrm{mA}$ to $100 \mathrm{~mA} ; 5 \mathrm{~mm}$ thick source images and the $1.25 \mathrm{~mm}$ reconstructed images. All CT examinations were interpreted on advantage workstation with $3 \mathrm{D}$ reconstruction and multiplanner reformation capability. Unenhanced low dose MDCT findings were categorised into:

i) Ureteric calculus detected.

ii) No ureteric calculus detected. This category included those with alternate diagnosis and negative scans.

Methods of reference: The subjects were followed up to determine if a ureteric calculus was present or by using a composite reference standard validated in previous studies $(5,7,8)$. Ureteral stone was considered to be definitely present if it was surgically retrieved or fragmented with ESWL, depicted on subsequent imaging studies showing evidence of calculus migration or stone excreted followed by relief of pain associated with macroscopic and microscopic haematuria. Ureteral stone was considered definitely absent on CT findings of alternative diagnosis explaining patient's symptoms, depiction of absence of ureteral stone or obstruction by subsequent imaging studies, negative microscopic urinalysis and relief of pain with no treatment and a laboratory-based alternative diagnosis (e.g., urinary tract infection), with complete resolution of symptoms.

Sample size and statistical analysis: Estimated sample size of 104, was calculated using the equation applied in descriptive studies designed to measure a characteristic in terms of proportion (10). This was on assumption that $90 \%$ of ureteric calculi would be detected with a confidence interval of $\pm 10 \%$ and a significant $\mathrm{p}$-value of 0.05 .

Analysis was carried out using Statistical Programmefor SocialSciences (SPSS 15.0) to calculate proportion of correctly diagnosed ureteric calculus and chi square test.

\section{RESULTS}

Onehundred and four patientsunderwentunenhanced low dose MDCT KUB, 90 (47 male and 43 female with age range of 17 - 79 years, mean 40 and SD 11.5) were included in the statistical analysis. Fourteen were excluded for not meeting the inclusion criterion.

Eighteen out of ninety (20\%) patients had ureteric calculi and 72/90(80\%) did not have ureteric calculus on the reference standard (Table 1). Of the 18 patients with ureteric calculus, 17 were correctly identified on unenhanced MDCT KUB, giving a proportion of $94.4 \%$ (17/18) (Table 3).

Among the 72 without ureteric calculus on the reference standard, 70 did not have any ureteric calculus unenhanced low dose MDCT giving a proportion of $97 \%$. twenty nine out of ninty (32\%) had alternative diagnosis made on unenhanced low dose MDCT (Table 2).

Table 1

Composite reference standard findings

\begin{tabular}{ll} 
Ureteric calculus definitely present & 18 \\
No ureteric calculus present & 72 \\
\hline Total & 90 \\
\hline
\end{tabular}

Table 2

Unenhanced low dose MDCT findings

Unenhanced low dose No. $\quad(\%)$

MDCT KUB findings

\begin{tabular}{lcc}
\hline Ureteric calculi & 19 & 21 \\
No ureteric calculi & & \\
Alternate diagnosis & 29 & 32 \\
Negative scans & 42 & 47 \\
\hline Total & 90 & 100 \\
\hline
\end{tabular}

The total number of positive unenhanced low dose MDCT KUB (ureteric calculus and alternate diagnosis) was $48 / 90(53 \%)$.

Table 3

Unenhanced low dose MDCT KUB compared with reference standard with respect to ureteric calculi

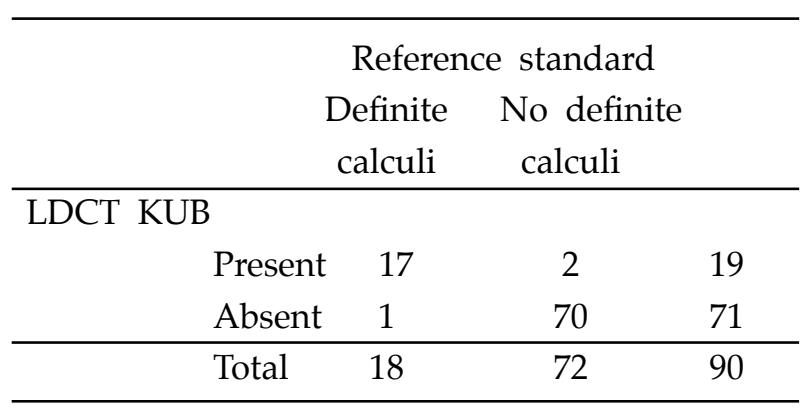


Figure 1

Axial and reformatted coronal images at $100 \mathrm{mAs}$ for a 39 year female with $10 \mathrm{~mm}$ right vesicoureteric calculus with proximal hydroureter, hydronephrosis and perinephric stranding

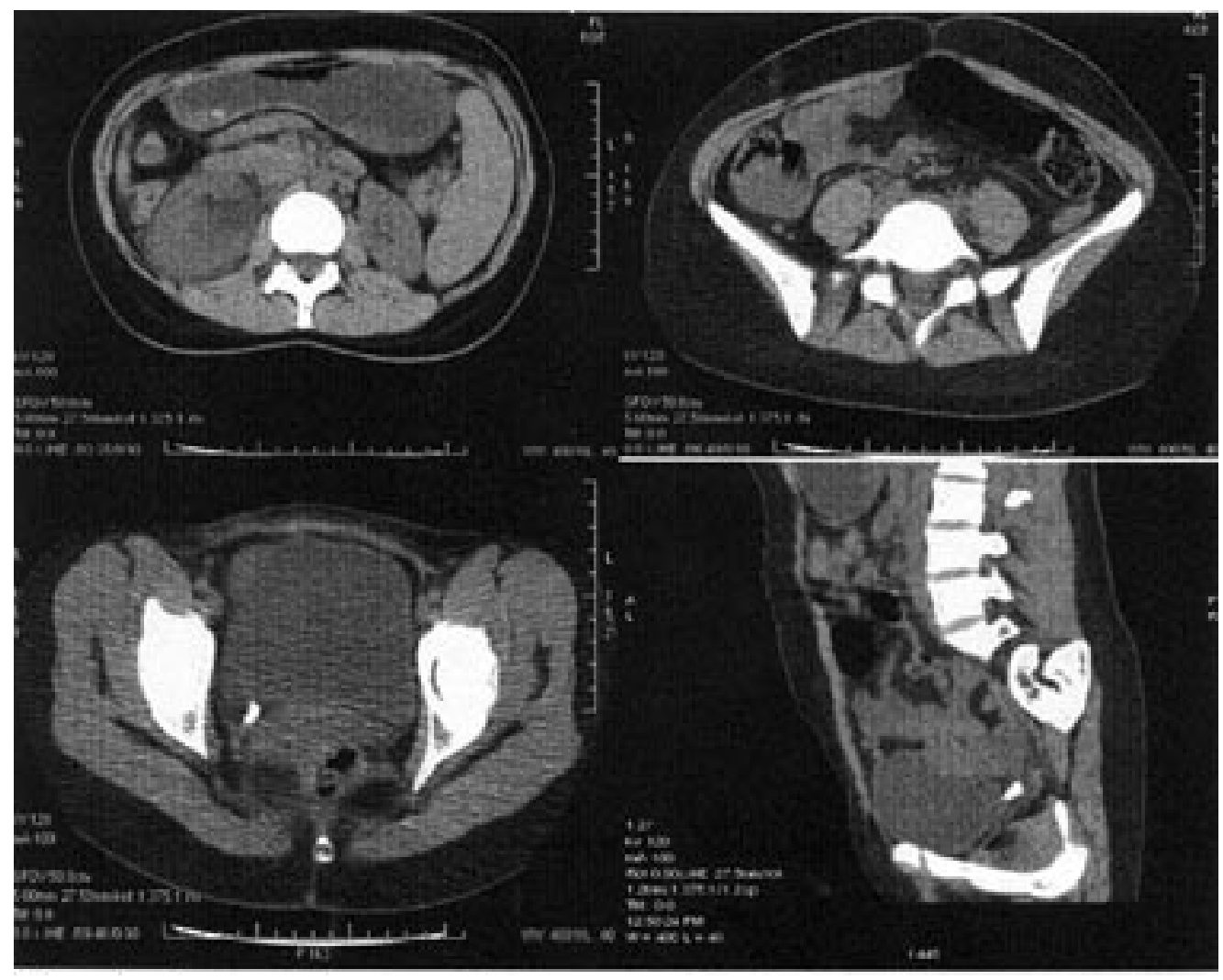

Figure 2

Axial and reformatted oblique views of a 49 year male with $7.5 \mathrm{~mm}$ right ureteric calculus; this was confirmed by ureteroscopy

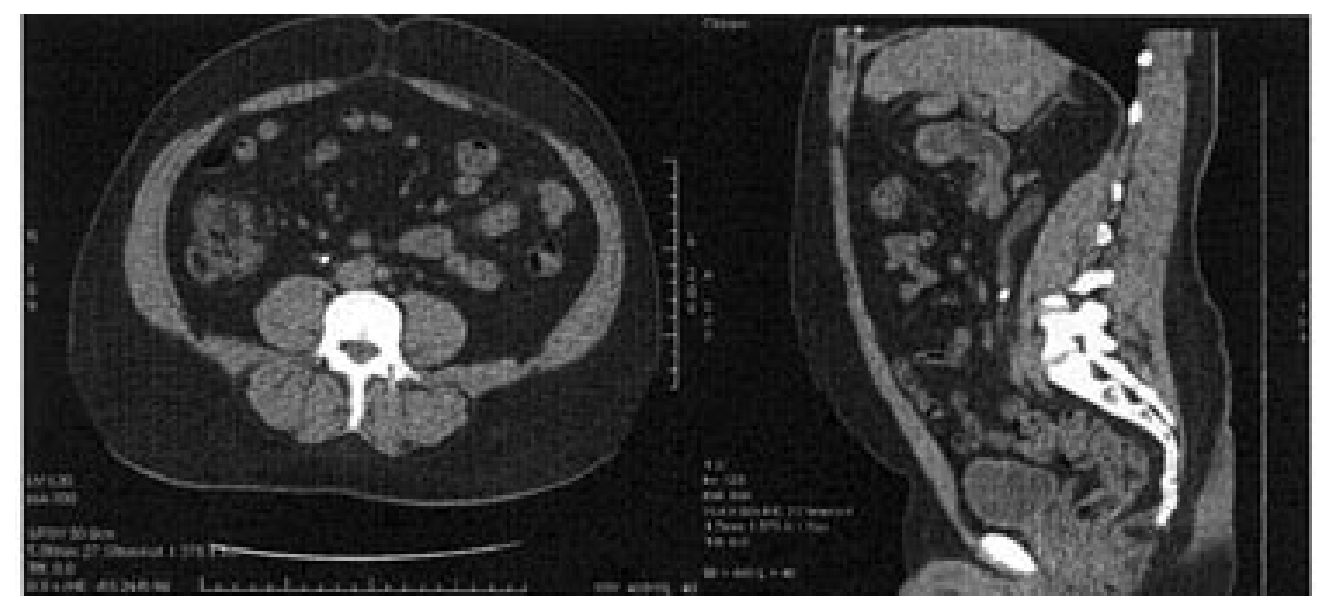




\section{Figure 3}

$A$ and $B$ are $L D C T$ axial images of a 58 year diabetic, demostrating perinephric collection with air fluid level consistent with emphysematous pyelonephritis. $C$ and $D$ are post-surgical images showing improvement with drain in situ. Multi-drug resistant E. coli was cultured

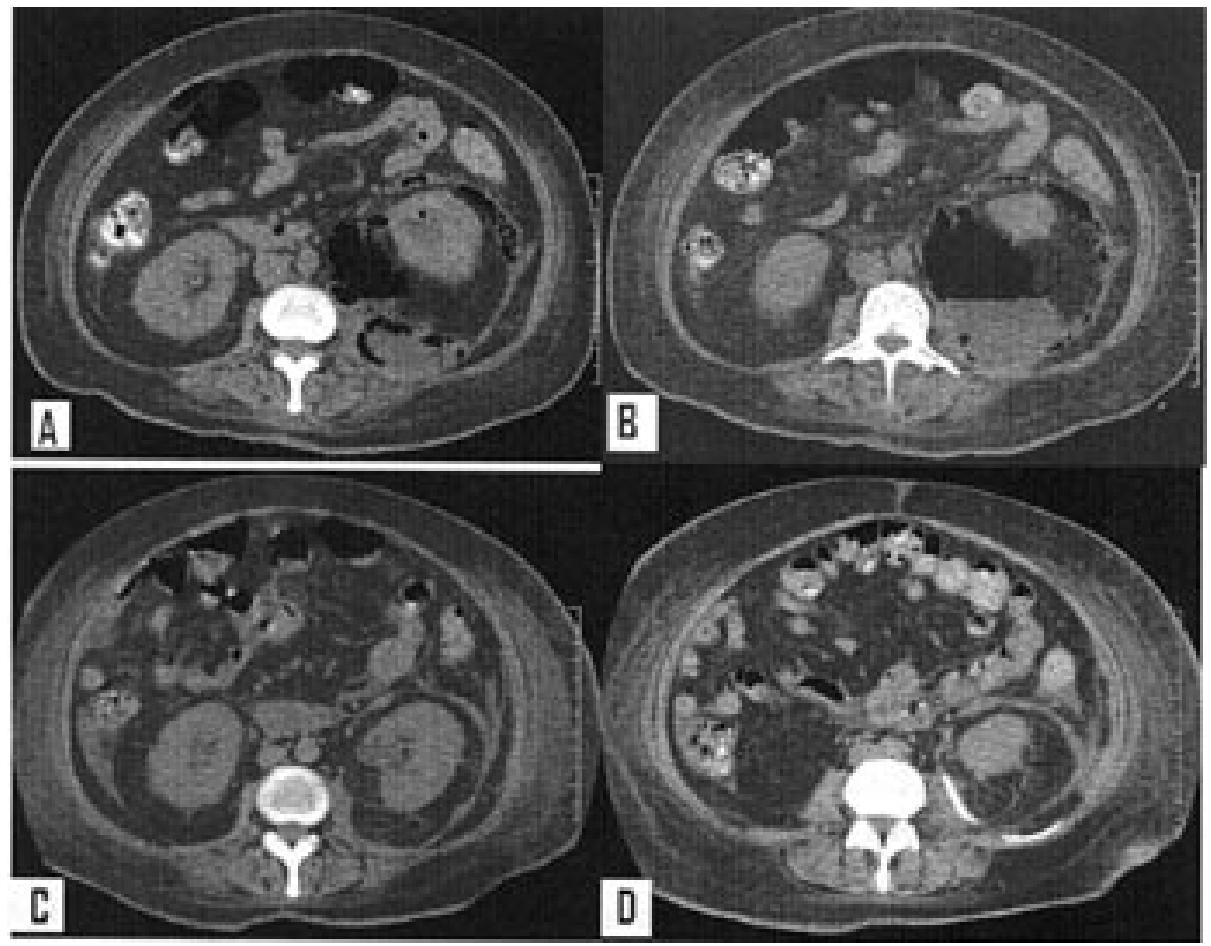

Figure 4

LDCT KUB axial images of a 54 year male demonstrating fine increased attenuation of mesenteric fat (arrows) consistent with mesenteric panniculitis possibly due to mild pancreatitis. He improved on conservative management

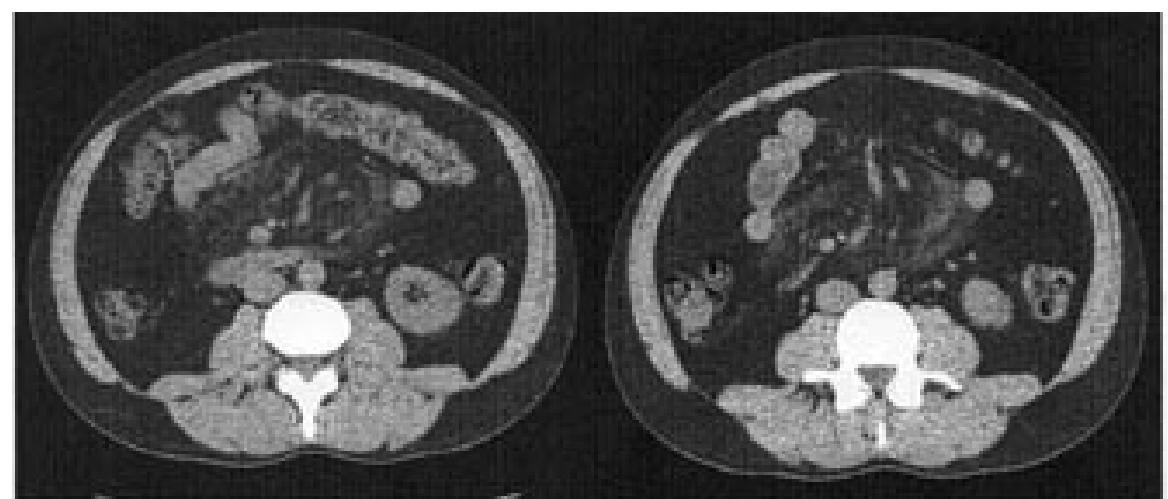

DISCUSSION

The study demonstrates that unenhanced low dose MDCT is the preferred of examination for evaluation of renal colic patients as evidenced by the high proportion of correctly diagnosed ureteric calculus $17 / 18(94.4 \%)$.

An alternate diagnosis was detected in 29(32\%) of the total patients. This has been reported to range from 30 to $38 \%$. The spectrum of alternate diagnoses detected in this population is similar to that reported in the literature. These included among others: unilateral periureteric focal mass of retroperitoneal fibrosis, mesenteric panniculitis, breast vertebral metastasis and tuberculosis of spine presenting as renal colic. Though there were more patients with alternative diagnosis than those with ureteric calculus, CT KUB is considered positive if either is found. This further confirms the reversed trend noted by Chen et al (11) where a decrease from 49 to $28 \%$ in the rate of detected obstructing stones in the ureter and a corresponding increase from 16 to $49 \%$ in alternate diagnosis rate were documented. Though renal colic pain due to solely suspected ureteric calculi is the only justified 
indication, Chen et al (11) argued that this reversal was due to the broadened indication for the CT KUB. This broadening of criteria to include non-specific lumbar pain leading to unenhanced CT may be also explained in partby the lower cost of the examination compared to the standard abdominal and pelvis CT with oral and IV contrast. Also noted was the increased awareness by the referring physicians of the value of unenhanced CT in finding an abnormality or establishing the source of the patient's symptoms.

The total number of patients with ureteric calculi in this study was however low (20\%), largely due to poor patient selection and tendency to request for this examination for almost any patient presenting with loin or flank pain. These two factors have also been described in the literature (12). In addition the ureter has a similar anatomical innervation with other visceral organs which when diseased can cause renal colic pain; therefore it's uncommon to have confusion about the source of the pain both to the physician and patient in the emergence department.

There was no patient who underwent a high dose after the low dose MDCT KUB for any reason, which further emphasizes the fact that unenhanced low dose MDCT was satisfactory to the reporting radiologist in evaluation of renal colic patients in this study group. The other reason could be the post acquisition processing capability on the reporting workstation which enabled multiplanner and 3D reconstruction and therefore enhanced confidence during interpretation. Follow up standard CT abdomen was recommended in only 14/90(15\%) mostly for the sole purpose of further characterisation and evaluation of an already suspected alternate diagnosis. Approximately $12 \%$ of the studies usually proceed to standard CT abdomen as reported in literature (12). One patient proceeded to have CT urography for what was discovered later as a malignant ureteric stricture.

The only parameter adjusted in this study was reduction of $\mathrm{mA}$ from an average of 200 to 100 . Since radiation exposure is directly proportional to the $\mathrm{mA}$ this meant that radiation exposure and dose were reduced to a half, which was the major goal of the study. Radiation risk was therefore reduced without compromising diagnostic performance of the examination and final outcome of the results.

MDCT KUB protocol of 180 - 280mA (120$140 \mathrm{kVp})(4)$ is considered to be the gold standard for evaluation of ureteric calculi; however it would be costly and unethical to radiate the same population twice. Finding of a single alternate reference standard all patients remains unclear as also noted by Douglas, et al (3). However surgical retrieval of the ureteric stone and actual visualisation of a passed sieved stone remain unchallenged outcomes but not all patients could be subjected to this. That is why a composite reference standard was used to validate the LDCT findings.

The advantages of this protocol over IVPinclude its speed, non utilisation of contrast to patients who may already have compromised renal function, high accuracy for diagnosis or exclusion of stone and determination of stone burden and size and location.

Single detector spiral CT protocol at reduced radiation dose can be used such as that Hamm et al (13) reported in their study with high sensitivity and specificity.

International Committee on Radiation Protection (ICRP) recommendation is based on the ALARA principle, which stands for "As low as reasonably achievable". The outcome of the study also provides evidence that this reduced radiation dose protocol of MDCT, can be applied in African population presenting with renal colic without compromising diagnostic performance.

In this study, low dose multidetector CT KUB can be effectively used for evaluation of suspected renal colic patients as demonstrated by the statistically significant correctly detected ureteric calculi.

\section{ACKNOWLEDGEMENTS}

To the AKU research support unit, Drs. U. Usman, S. Vinayak, A. Zafar, S. Samnaky, S. Naji, D. Gitau, K. Maganda and P. Osire and B. Okoo for the help and support during this research project.

\section{REFERENCES}

1 Kevin K., Smith, et al. Emedicine: Nephrolithiasis/ Urolithiasis. Last Updated: November 4, 2005

2 Weldon, Liu., Stephen, J. E., Brian J. K. et al. Low-dose non-enhanced helical CT of renal colic: Assessment of ureteric stone detection and measurement of effective dose equivalent. Radiology. 2000; 215:51-54.

3 Douglas, S. Katz., et al. Commentary: Can low-dose unenhanced multidetector CT be used for routine evaluation of suspected renal colic? Amer. J. Roent. 2003; 180: 313-315.

4 Denis T., Stavroula, S., Isabelle, D. et al. Low-dose unenhanced multidetectorCT of patients withsuspected renal colic. Amer. J. Roent. 2003; 180: 305-311.

5 Smith, R.C., Rosenfield, A.T., Choe, K.A., et al. Acute flank pain: comparison of non-contrastenhanced CT and intravenous urography. Radiology. 1995; 194: 789 -794.

6 Sommer, F.G., Jeffrey, R.B. Jr., Rubin, G.D., et al. Detection of ureteral calculi in patients with suspected renal colic: value of reformatted no contrast helical CT. Amer. J. Roent. 1995;165:509 -513. 
7 Smith, R.C., Verga, M., McCarthy, S. and Rosenfield, A.T. Diagnosis of acute flank pain: value of unenhanced helical CT. Amer. J. Roent. 1996; 166: 97 -101 .

8 Sourtzis, S., Thibeau, J.F., Damry, N., et al. Radiologic investigation of renal colic: unenhanced helical CT compared with excretory urography. Amer. J. Roent. 1999; 172:1491 -1494.

9 Poletti, P.A., Platon, A., Rutschmann, O.T., et al. Lowdose versus standard-dose CT protocol in patients with clinically suspected renal colic. Amer. J. Roent. 2007; 188: 927 -933

10 John Eng. Sample size estimation: How many individuals should be studied? Radiology. 2003; 227: 309-313.
11 Chen, M.Y., Zagoria, R.J., Sauders, H.S., et al. Trends in the use of unenhanced helical CT for acute urinary colic. AJR. 1999;173: 1447-1450.

12. Lee, J.K., Hiken, I.N. and Semelka, R.C. The kidney and ureter. In: Computed body tomography with MRI correlation. Philadelphia, PA: Lippincott Raven, 2006; 1242

13. Hamm, M., Knopfle, E., Wartenberg, S., et al. Low dose unenhanced helical computerized tomography for the evaluation of acute flank pain. J. Urol. 2002;167: $1687-1691$. 